- A basic pack of all the endodontic instruments required must be available suitably sterilised to ensure rapid efficient treatment.

- Modern radiographic techniques facilitate swift diagnosis and treatment procedures.

- The development of endodontic instruments from reamers to greater taper nickel titanium files is considered.

- The importance of thorough and efficient irrigation with appropriate antiseptic agents is discussed, together with the necessary precautions.

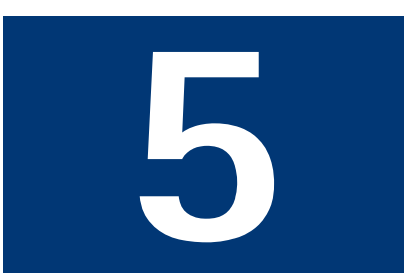

\title{
Endodontics: Part 5 Basic instruments and materials for root canal treatment
}

\section{P. Carrotte ${ }^{1}$}

In this part the basic endodontic instruments necessary for effective root canal treatment are described. The properties of, and manufacturer's claims for, new instruments and techniques may be compared to these basic principles before they are purchased and introduced to clinical practice. Having the correct instruments for different clinical situations may make treatment both more efficient and more effective.

\section{ENDODONTICS}

1. The modern concept of root canal treatment

2. Diagnosis and treatment planning

3. Treatment of endodontic emergencies

4. Morphology of the root canal system

5. Basic instruments and materials for root canal treatment

6. Rubber dam and access cavities

7. Preparing the root canal

8. Filling the root canal system

9. Calcium hydroxide, root resorption, endo-perio lesions

10. Endodontic treatment for children

11. Surgical endodontics

12. Endodontic problems

${ }^{1 *}$ Clinical Lecturer, Department of Adult Dental Care, Glasgow Dental Hospital and School, 378 Sauchiehall Street, Glasgow G2 3JZ

${ }^{*}$ Correspondence to: Peter Carrotte Email:p.carrotte@dental.gla.ac.uk

\section{Refereed Paper}

doi:10.1038/sj.bdj.4811738

๑ British Dental Journal 2004; 197:

455-464
Many dental practitioners find it difficult to resist new gadgets, and there are an inordinate number made specifically for endodontics. New instruments and materials are frequently sold with the promise of simplifying a technique, shortening the time taken or even increasing the success rate. Unfortunately, these promises are often not fulfilled, and the result may be cupboards in the practice containing unwanted endodontic armamentaria. It would be impossible to cover all the instruments and materials used in endodontics in one part, but it is hoped to mention most of the basic equipment and discuss some of the newer items. For continuity, some instruments will be described in the relevant parts. The majority of the instruments and materials referred to in this part are generic, and may be purchased from most dental supply companies.

\section{INSTRUMENT PACK}

A basic pack of instruments must be available specifically for routine root canal procedures. An example is given in Figure 1. A front surface reflecting mouth mirror is preferable to prevent the double image of the fine detail in an access cavity that occurs with a conventional mirror. Endolocking tweezers allow small items to be gripped safely and passed between nurse and operator. A DG16 endodontic probe is required to detect canal orifices. The excavator is long shanked, with a small blade to allow access into the pulp chamber. The pocket-measuring probe is useful, a routine CPITN probe with clearly vis-

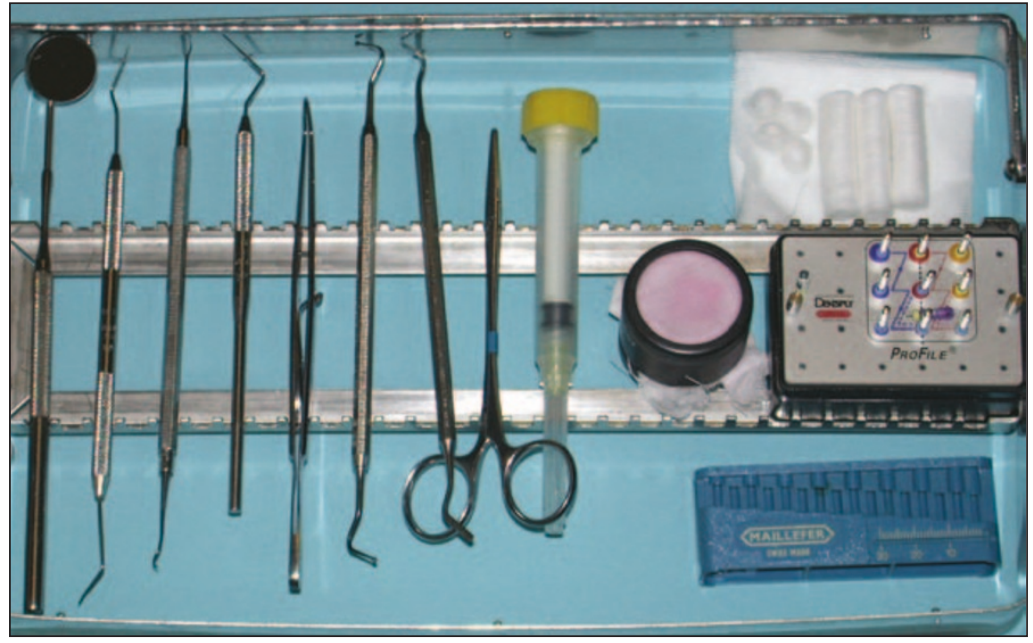

Fig. 1 An endodontic instrument pack. From left to right; front surface reflecting mirror; DG16 endodontic probe; Western probe; CPITN probe; endo-locking tweezers; long shank excavator; flat plastic, artery forceps, endodontic syringe; plus clean stand, file stand, measuring device, sterile cotton wool rolls and pledgets. 


\section{PRACTICE}

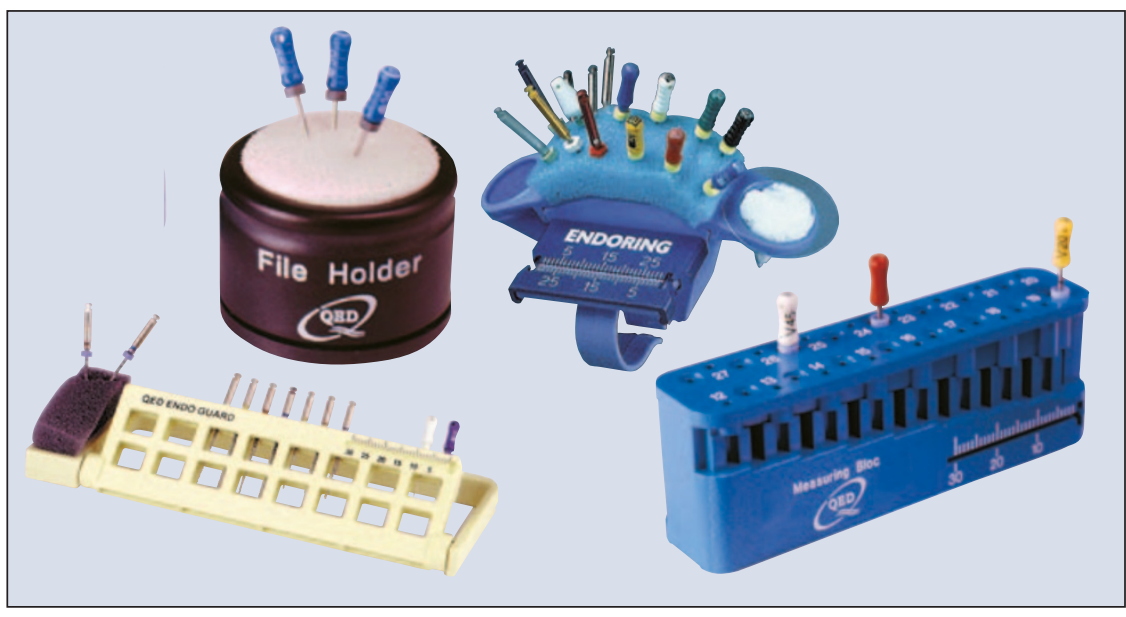

Fig. 2 A selection of file holders.

Fig. 4 The rubber dam equipment; clamps, dental floss, forceps, sheet, punch, frame and napkin.

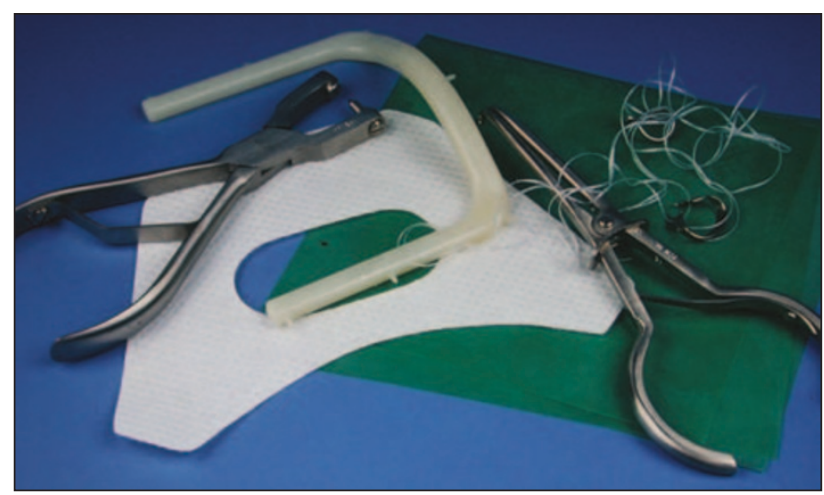

ible gradations is ideal. A furcation probe is useful to check for the presence of furcation involvement. Other items usually included are a flat plastic, sterile cotton wool rolls, sterile cotton wool pledgets, artery forceps to grip a periapical radiograph and a metal ruler, or other measuring device that may be sterilized. A clean-stand or other device such as the endoring is required to hold the endodontic instruments. Paper points are also required, and the simplest method of storage and use is to purchase presterilized packs with five points in each pack.

These instruments should be sterile when treatment commences, and every possible effort must be made to avoid contamination. Few practices will have an autoclave sufficiently large to take a metal tray with a lid that may contain an entire set of sterile instruments. If an open tray system is used, as illustrated in Figure 1 , it is useful to have all endodontic instruments in sterilized containers, such as the clean stand or endodontic ring shown in Figure 2. This allows the instruments to be easily controlled, and accessed by both the operator and assistant during treatment.

\section{PATIENT PROTECTION}

Glasses are needed to protect the patient's eyes. Figure 3 also shows a waterproof bib being worn, as the patient's clothes must be protected against accidental spillage of sodium hypochlorite, a frequent source of patient complaint or even litigation.

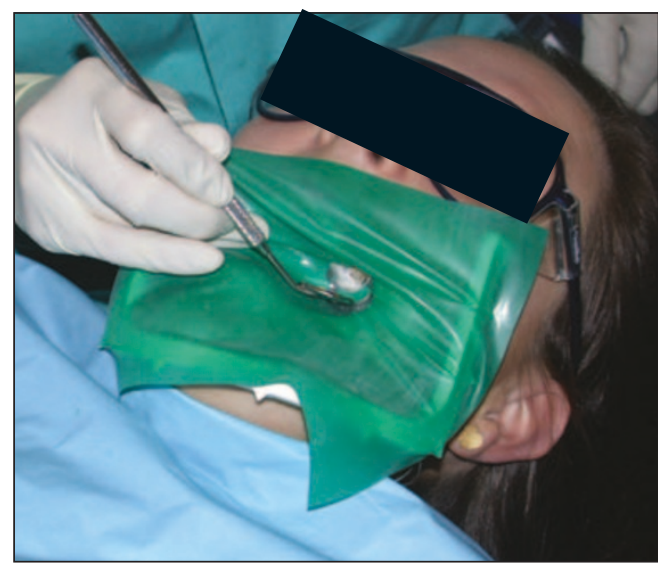

Fig. 3 A patient wearing safety glasses, a waterproof protective bib and a rubber dam.

\section{RUBBER DAM}

Rubber dam is essential in root canal treatment for three reasons:

- To provide an operating field free from oral contamination.

- To prevent the patient swallowing or inhaling root canal instruments or medicaments.

- To give good visual access by retracting the lips and tongue.

A basic kit for rubber dam equipment is shown in Figure 4. Details of this equipment, and of the techniques for the application of rubber dam, are given in the next part.

\section{RADIOGRAPHIC EQUIPMENT}

Long-cone parallel radiography is a requirement for endodontics, ${ }^{1}$ because it gives an undistorted view of the teeth and surrounding structures and is repeatable, thus allowing more accurate assessment of periapical healing. The bisecting angle technique should no longer be employed. It is further recommended that rectangular collimation
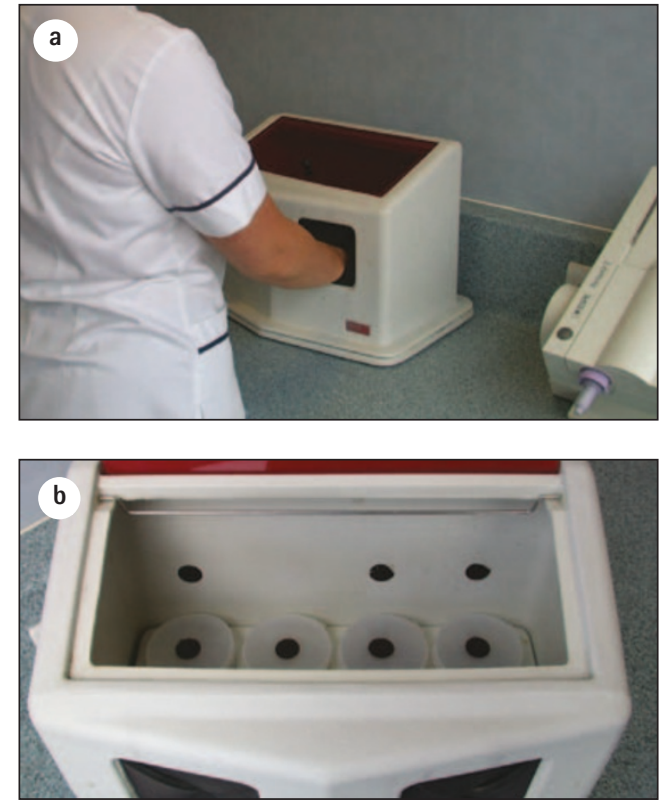

Fig. 6 a) A manual radiographic processing unit being used and b) containing rapid developing and fixing chemicals. 


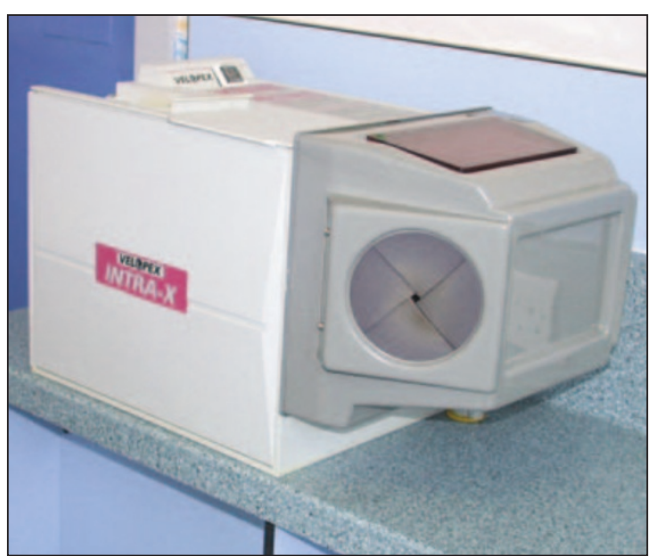

Fig. 7 An automatic radiographic processor, with a simple device to bypass the drying cycle.

be fitted on all new radiographic equipment, and retro-fitted to existing equipment as soon as possible. There are many beam-aiming devices available to hold the x-ray film parallel to the tooth. Figure 5 shows an example of a popular holder, with a special cage attachment to fit over a rubber dam clamp.

A quick, reliable method of viewing radiographic images is essential for endodontics. Considerable time can be lost if such a system is not available, especially on those occasions when the exposed film does not show the required detail. Practitioners using conventional radiography may wish to purchase an x-ray film processor with rapid developing and fixing solutions, capable of producing a radiograph for viewing in under a minute; an example is shown in Figure 6. However, a modern automatic processor (Fig. 7) may be adjusted to deliver wet films in under two minutes. Films from both types of processor should be carefully dried after viewing for accurate storage in the patient's records.

A modern alternative involves the use of digital radiography. A sensor plate, appropriately sterilized and sealed, is used in place of the conventional film. The sensor may be either directly linked to the computer, or resemble a conventional periapical film packet. The resultant image is digitally processed and projected upon the computer screen in a matter of seconds. The quality of the image can be manipulated to enable greater clarity when reading the picture. For purposes of record keeping, the image may be either dated, labelled and stored in the central database, or a hard copy printed for the patient's records. An example of such a system, and the images produced, is shown in Figure 8.

The pre-operative radiograph contains much information to assist the operator, which may not be seen if the film is carelessly viewed. An xray viewer and some form of magnification are needed to examine periapical films, and it is very helpful if glare from the light around the radiograph can be excluded (Fig. 9).

\section{DEVELOPMENT OF HAND INSTRUMENTS}

For many years the standard cutting instruments have been the reamer, K-type file and Hedstroem
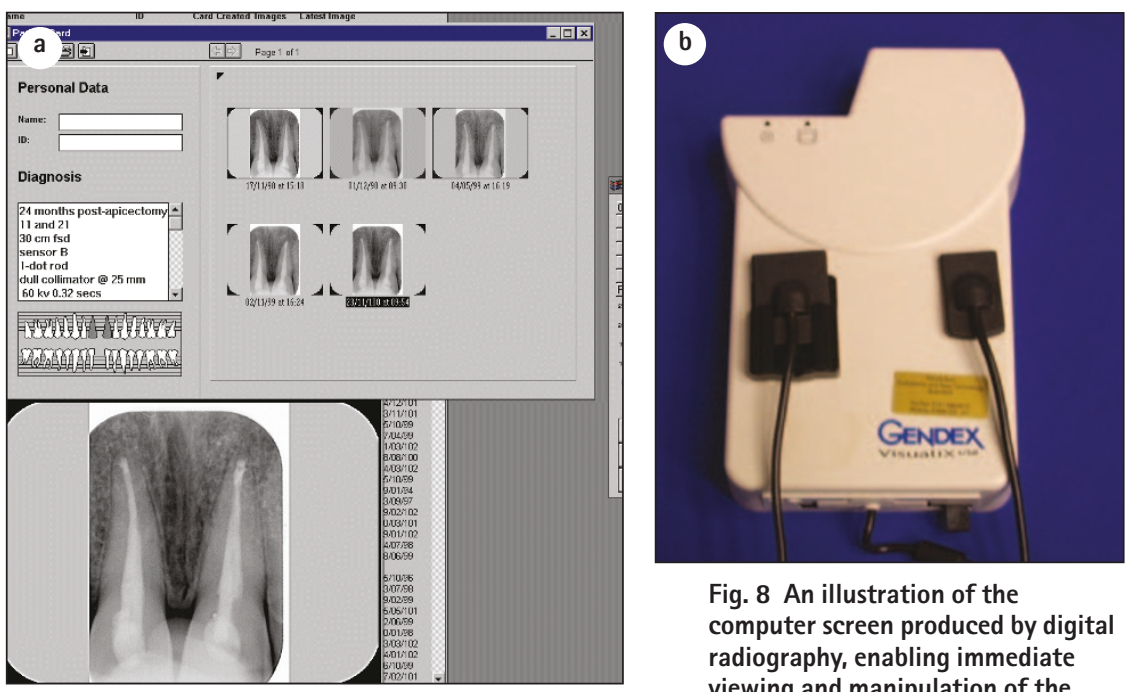

Fig. 8 An illustration of the computer screen produced by digital radiography, enabling immediate viewing and manipulation of the image The sensor plate or 'film' may be either loose, resembling a

file. These root canal preparation instruments have been manufactured to a size and type advised by the International Standards Organisation (ISO). The specifications recommended are complex and differ according to the type of instrument. For most standardized instruments the number refers to its diameter at the tip in one-hundredths of a millimetre; a number 10 , for example, means that it has a tip diameter of $0.10 \mathrm{~mm}$. Colour coding originally denoted the size, but now represents a sequence of sizes. All these instruments have a standard 2\% taper over their working length.

Recent changes in both metallurgy and endodontic concepts have led to the introduction of a range of new instruments which do not conform to these specifications. These are described individually later and in Part 7. These instruments have been widely adopted, and appear to give consistently better results in root canal treatment. However, the conventional 2\% taper instruments are essential for the initial exploration of most root canals, for difficult procedures such as bypassing separated instruments, and for the apical preparation of some difficult canals.

Conventional 'standardized' instruments are made of steel, which may wear quickly in dentine, and small size files may be regarded as disposable. Although some hand files are now available in a nickel-titanium alloy, which is more resistant to wear than ordinary steel, the increased cost and inability to pre-curve has not led to their widespread use. The majority of these modern files are manufactured with a modified non-aggressive tip to prevent iatrogenic damage to the canal system, and improve performance of the instrument. Figure 10 shows the different appearance of the principal types of these instruments.

\section{K-type file}

These instruments were originally made from a square or triangular blank, machine twisted to form a tight spiral. The angle of the blades or flutes is consequently near a right-angle to the shank, so that either a reaming or a filing action

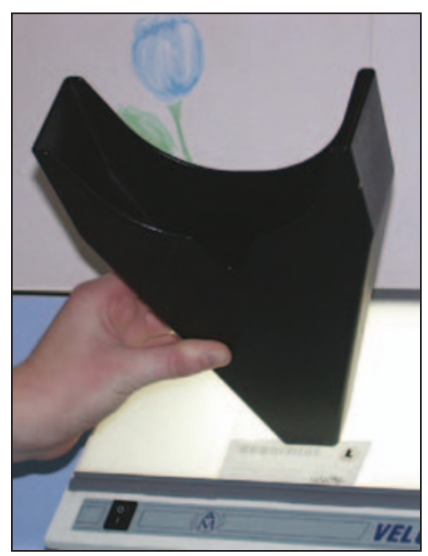

Fig. 9 A radiographic viewer designed to eliminate extraneous light and magnify the image.

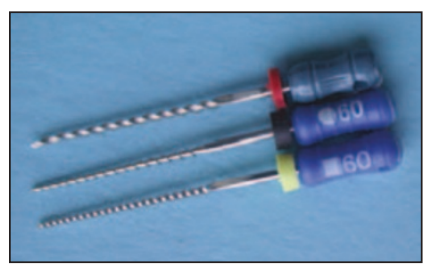
instruments; top - reamer with red stop; middle - Hedstroem file with black stop; bottom - K-flex file with yellow stop.
Fig. 10 Conventional hand 


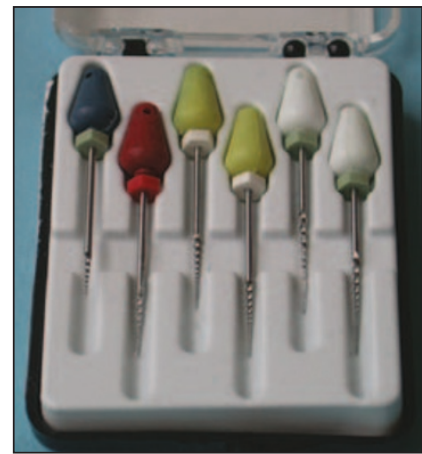

Fig. 11 A pack of hand files of greater taper, $12 \%$ taper (blue), 10\% (red), $8 \%$ (yellow) and 6\% (white).

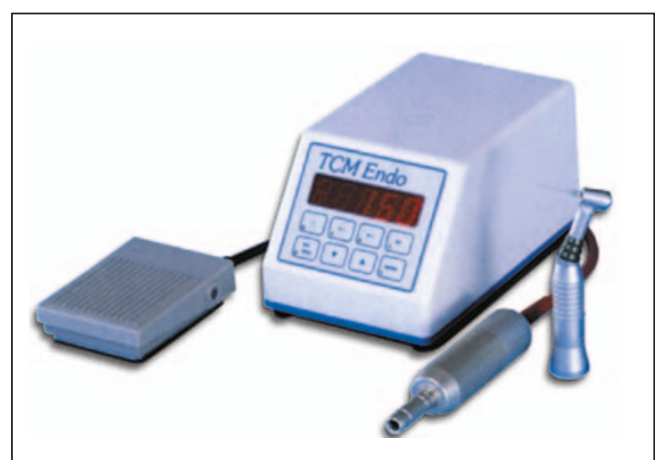

Fig. 12 A low-speed, high-torque motor required for use with nickel-titanium rotary instruments.

may be used. The K-type file has been subject to continuous development. The $\mathrm{K}$-flex file is made from a rhomboid or diamond shaped blank. The acute angle of this shape provides the instrument with two sharp blades and the narrower diameter allows greater flexibility in the shaft than a conventional K-file. The manufacturers claim that more debris is collected between the blades and therefore removed from the canal than with a standard K-file. The Flex-o-file employs a more flexible type of steel. It does not fracture easily and is so flexible that it is possible to tie a knot in the shank of the smaller sizes.

The latest developments in file design have seen a move away from the ISO standard 2\% taper to files with increasing tapers of up to $12 \%$, made in a nickel-titanium alloy. Although most of these new developments are used with an electric motor, hand files of greater taper are available. These are illustrated in Figure 11. Their use is described in Part 7.

Although most K-type files were originally used with an 'in-out' circumferential filing technique, the 'balanced-force' technique, described in Part 7, is now considered the manipulation method of choice.

\section{Hedstroem file}

The Hedstroem file is machined from a round tapered blank. A spiral groove is cut into the shank, producing a sharp blade. Only a true filing action should be used with this instrument because of the angle of the blade. There is a strong possibility of fracture if a reaming action is used and the blades are engaged in the dentine. The Hedstroem file is useful for removing gutta-percha root fillings.

\section{Other hand files}

Different types of hand file have been introduced from time to time with varied structure and cutting action. The Unifile and Helifile were modifications of the Hedstroem design. The Mani Flare file is made from a triangular blank, and features a greater taper than conventional $2 \%$ files. It is essential when considering the use of new file designs that the operator understands the basic principles of canal preparation, and compares and contrasts the properties with the file manipulation technique currently being used.

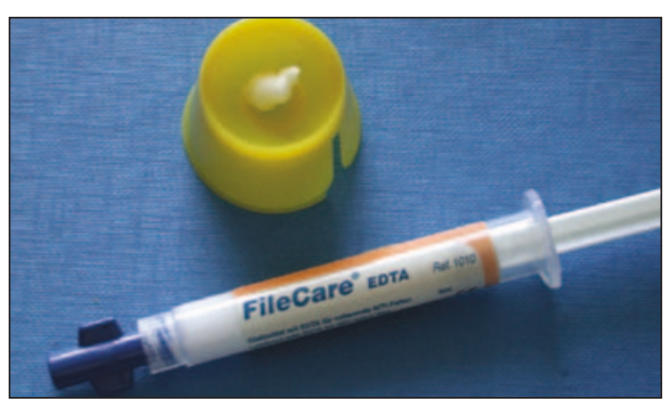

Fig. 13 An EDTA paste which will usually be picked up on each instrument before use.

\section{Reamer}

The reamer is constructed from a square or triangular blank, machine twisted into a spiral but with fewer cutting flutes than a file. The reamer will only cut dentine when it is rotated in the canal; the mode of action described for its use is a quarter to a half turn to cut dentine, and withdrawal to remove the debris. The stiffness of an instrument increases with each larger size, so that larger reamers in curved canals will tend to cut a wider channel near the apical end of the root canal (apical zipping). Considerable damage may be caused to a root canal by the incorrect use of a reamer, and their routine use is no longer recommended.

\section{Power-assisted instruments}

Handpieces providing a mechanical movement to the root canal cutting instrument have been available since 1964 . Their function was primarily a reciprocating action through $90^{\circ}$ and/or a vertical movement, according to the design and make. Because steel files do not have the flexibility necessary for rotary movements in a curved canal without damaging the canal configuration, these instruments were never really acceptable in endodontic practice.

A totally new concept in canal preparation came with the development of sonic and ultrasonically activated endodontic handpieces. Much research took place into the mode of action and effectiveness of these machines. It was generally agreed that while the sonic machines were more effective at hard tissue removal, the ultrasonic machines were more effective in irrigation. The piezo-electric machines were found to be more effective than the magnetostrictive. The latter also generated more heat, and irrigation with effective quantities of sodium hypochlorite was found to be difficult.

The ultrasonic action causes acoustic microstreaming of the irrigant, intensive circular fluid movement carried right to the tip of the instrument, found to be very effective at canal debridement. This effect is reduced, however, when the file is constrained by the canal wall. The main use of these instruments today is in irrigation and debridement, using a freely oscillating file in a sodium hypochlorite filled canal, after thorough mechanical canal shaping. ${ }^{2}$ 
However, the development of nickel-titanium alloy for endodontic instruments has allowed the concept of an engine driven endodontic instrument to be fully explored. The total flexibility of this alloy, and the use of radial lands on the cutting flutes to keep the instrument centred in the canal, permit controlled cutting of the dentine walls. Most major manufacturers have developed a nickel-titanium rotary system. Lightspeed, Profiles, GT Rotary files, FlexMaster, Quantec system, Hero, K3, Protaper, and no doubt more will appear before this book is even published. It would not be possible to describe each of these fully, but the basic concepts are presented here, with a general description of their use being given in Part 7.

The systems will generally conform to one of three patterns.

- The system may have a standard ISO tip size sequence, with the instruments being manufactured with an increased taper, usually either $4 \%$ or $6 \%$.

- The system may be presented with a single tip size, but with the sequence of file sizes having an increased taper of up to $12 \%$. In order to accommodate this taper in a narrow root canal, the diameter of the instrument is usually limited to $1 \mathrm{~mm}$, giving quite a short functional blade in the greater tapers.

- Both of these new developments may be combined into one system.

A low-speed, controlled-torque motor is necessary when using these instruments, as illustrated in Figure 12.

\section{Irrigation and lubrication materials}

It is generally accepted in endodontic practice that sodium hypochlorite is the most suitable solution for irrigation of the root canal system. Normal household bleach is approximately 5.5\% sodium hypochlorite solution, and this may be diluted with purified water up to five times to the operator's preference. Research has shown that the antibacterial effect is the same for a $0.5 \%$ and a $5.0 \%$ solution. ${ }^{3}$ However, the greater the dilution the less effective is the solution at dissolving organic debris in the root canal system.

Great vigilance is essential when using sodium hypochlorite, and practitioners must be aware of the risks and dangers involved in its use. Irrigation under pressure may force the solution through the apical foramen into the periradicular tissues, which may result in a rapid, painful and serious inflammatory response. The patient will be extremely distressed, and little can be done to relieve the situation which may take several days to resolve. Cases have also been reported where excess pressure on the syringe has resulted in the needle coming loose and hypochlorite spraying over the patient, operator and assistant. Protective goggles are essential for the patient and all staff. Clothing should also be protected. The defence societies have received claims from irate patients for damaged clothing following root canal treatment. The practitioner must have appropriate risk assessment procedures in place when such materials are incorporated into their clinical practice.

Chlorhexidine solution $0.2 \%$ has a similar antibacterial action, but will not dissolve the organic debris found in parts of the canal system inaccessible to hand instrumentation, such as lateral canals, fins and apical deltas. However, the substantivity associated with this irrigant means that it will adhere to dentine, thereby exhibiting a prolonged antibacterial activity. Although chlorhexidine may not be quite as effective as sodium hypochlorite, its use should not be dismissed.

Researchers are constantly seeking improved methods of cleaning root canals; reports have appeared recently relating to the use of electroactivated water as an irrigant, ${ }^{4}$ and the use of high frequency electric current. ${ }^{5}$ These and others may prove interesting developments in root canal preparation and irrigation.

EDTA paste (Ethylenediamine tetra-acetic acid) is a chelating agent which softens the dentine of the canal walls and greatly facilitates canal preparation (Fig 13). EDTA solution may be used as an irrigant at the end of the canal preparation phase to assist removal of the smear layer prior to placement of an intervisit dressing, or obturation.

\section{Burs}

Several types of bur may be required for root canal treatment. Some of these are described below, and shown in Figure 14.

\section{Cutting an access cavity}

It is generally accepted that high speed burs should be used to gain access and shape the cavity. A diamond or tungsten carbide tapered fissure bur is used for initial penetration of the roof of the pulp chamber. A tapered safe-ended diamond or tungsten carbide bur is then used to remove the roof of the pulp chamber without damaging the floor.

\section{Location of canal}

Burs should only be used as a last resort to locate a sclerosed canal because of the danger of perforation. Small round burs are used; the standard length is usually too short but longer shank burs are available. Specially designed ultrasonic tips may also be used to remove secondary dentine, assist in the identification of canal orifices and in shaping the canal orifice during preparation. The use of ultrasonic tips has become more widespread with the introduction of a wider range of fittings to different piezo-electronic machines. Figure 15 shows the diamond coated CPR ${ }^{\circledR}$ tips, designed for troughing and chasing sclerosed canals, and the BUC ${ }^{\circledR}$ tips, with variable grades of diamond grit for refining access cavity walls and line angles, removing obstructions and cutting around posts. As with all instruments and materials, the manufacturer's instructions and guidance should be carefully

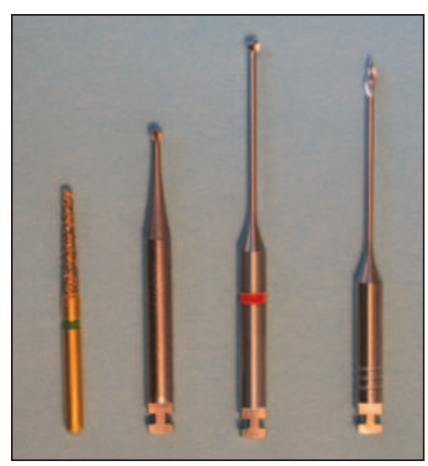

Fig. 14 Some of the burs specifically manufactured for endodontic treatment; a safe-tipped access bur; a long-shanked round bur; a swannecked bur; a Gates-Glidden bur. 


\section{PRACTICE}

Fig. 15 (a) CPR $^{\circledR}$ ultrasonic tips, now available to fit different piezoelectronic machines. (b) Also shown are KiS tips for periradicular surgery.

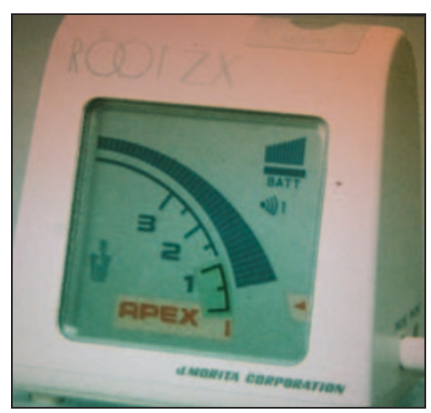

Fig. 16 An electronic apex locator.

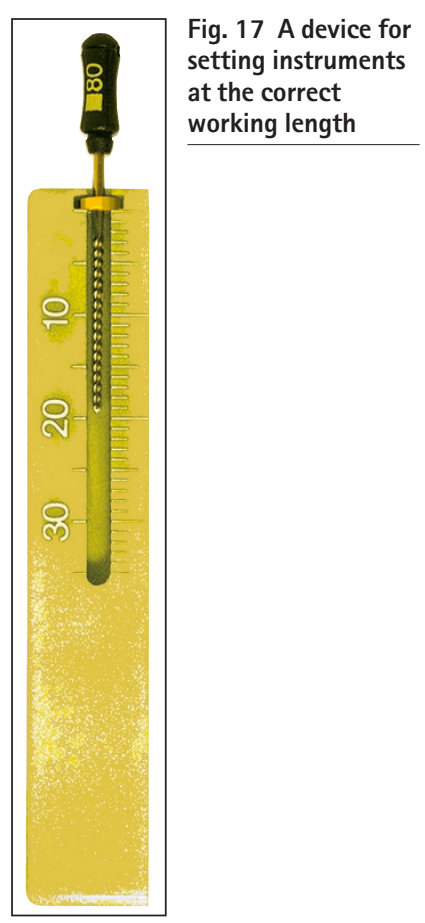

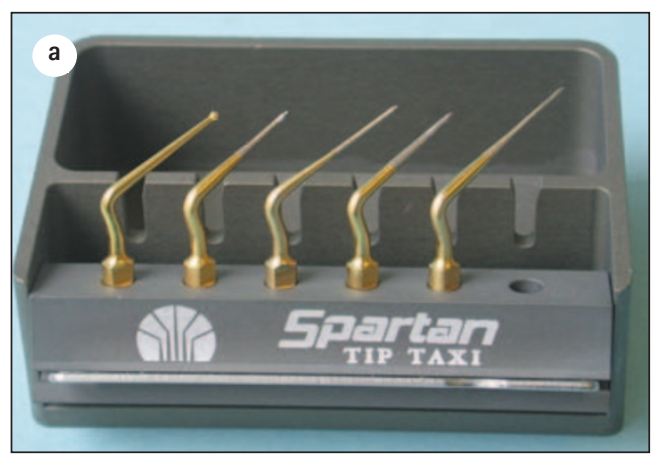

followed or these delicate diamond tips may be damaged. It is generally wise to use them with a low power setting, and to ensure that they are in contact with dentine before activating the piezoelectric unit.

\section{Canal preparation}

The use of rotary cutting instruments in a standard handpiece is condemned because of the danger of fracture of the instrument or perforation of the root canal. The exception to this rule is the Gates-Glidden bur, which has a safeended tip. In addition, the site of fracture, if it does occur, is almost always near the hub so the fractured piece is easily removed. In the past this bur has been recommended for initial flaring of the coronal portion of the canal. This may now be carried out in a more controlled manner with a nickel-titanium orifice shaper. The GatesGlidden bur may also be used to make post space and to remove gutta-percha from the canal. Gates-Glidden burs are manufactured in six sizes; their use is described in Part 7.

\section{Measurement of working length}

There are two established methods of assessing the working length of a root canal: one by radiography and the other with the use of an electronic device apex locator (Fig. 16). Both methods will be described in Part 7.

Once the working length has been confirmed, the individual preparation instruments must be accurately marked to length accordingly. There are many different gadgets available for transfer of the working length; the author prefers the device shown in Figure 17. There are also different stops for the instrument, the most popular being rubber or silicone stops. These should always be placed at right angles to the shank of the instrument. Ideally the stops should be either notched, or pear shaped, so that in curved canals the notch or point of the pear may be directed towards the curve placed in the instrument.

\section{Sterilization}

Any instrument which is placed in the root canal should be sterile, for two reasons. Firstly, to prevent the introduction to the root canal system of extraneous microorganisms, which may severely compromise treatment, for example pseudomonas. ${ }^{6}$ Secondly, if instruments and devices were to be used on different patients, to prevent cross-infection between patients. Bacte-

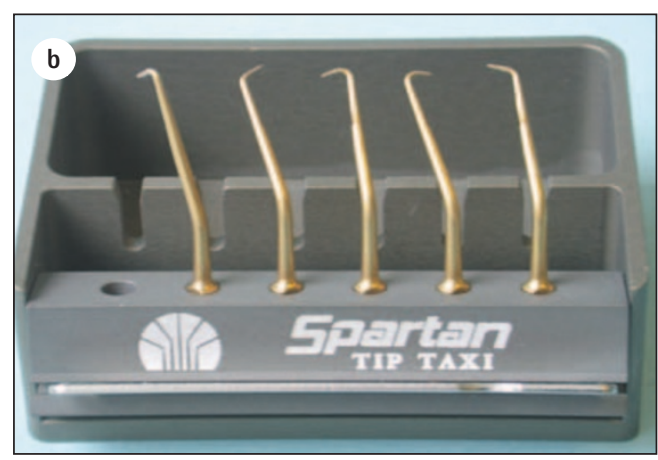

ria, viruses, fungi and prions may contaminate instruments and research has shown that some of these may not be destroyed by any method of sterilization. ${ }^{7}$ Figure 18 illustrates this dramatically. Concern has been raised over the sterilization of other items of dental equipment as well. ${ }^{8}$

Under the Medical Devices Directive, the manufacturer of any dental instrument has an obligation to inform the end-user (ie the dentist) how their product should be decontaminated. It is essential that this guidance is followed. Whatever may be written in this and other texts may be superseded at any time. Dentists should therefore ensure that they are familiar with and conform to the manufacturer's instructions. At present, some endodontic instruments are marked with the symbol shown in Figure 19 indicating that they are single use instruments. It is assumed that all manufacturers will shortly follow this Medical Devices Directive.

It may, however, be necessary to sterilize instruments for further treatment of the same patient on a subsequent occasion when crossinfection control would not be a problem. After use, instruments must be cleaned as soon as possible to remove debris which harbours and protects microorganisms. Cleaning is carried out by scrubbing in warm water and detergent, although the debris may be first removed from most root canal instruments by stabbing them into a sponge. The best method of cleaning is to place the instruments into an ultrasonic bath. The cavitational effects of ultrasonics will dislodge debris from places which are inaccessible to normal cleaning. When the instruments are clean they must be sterilized in an autoclave. Microorganisms are destroyed at lower temperatures and in a shorter period in moist heat as all biological reactions are catalysed in water. The disadvantages of autoclaving are that metal instruments tend to corrode and sharp instruments are dulled.

\section{Barbed broach}

This instrument has sharp rasps pointing towards the handle. They may be used to remove the contents of the root canal before commencing shaping procedures. A vital pulp may be extirpated when carrying out elective endodontic procedures, or when treating a tooth with an irreversible pulpitis, by introducing the barbed broach deep in the canal, twisting it a quarter to a half turn, and withdrawing, as shown in Figure 20. 

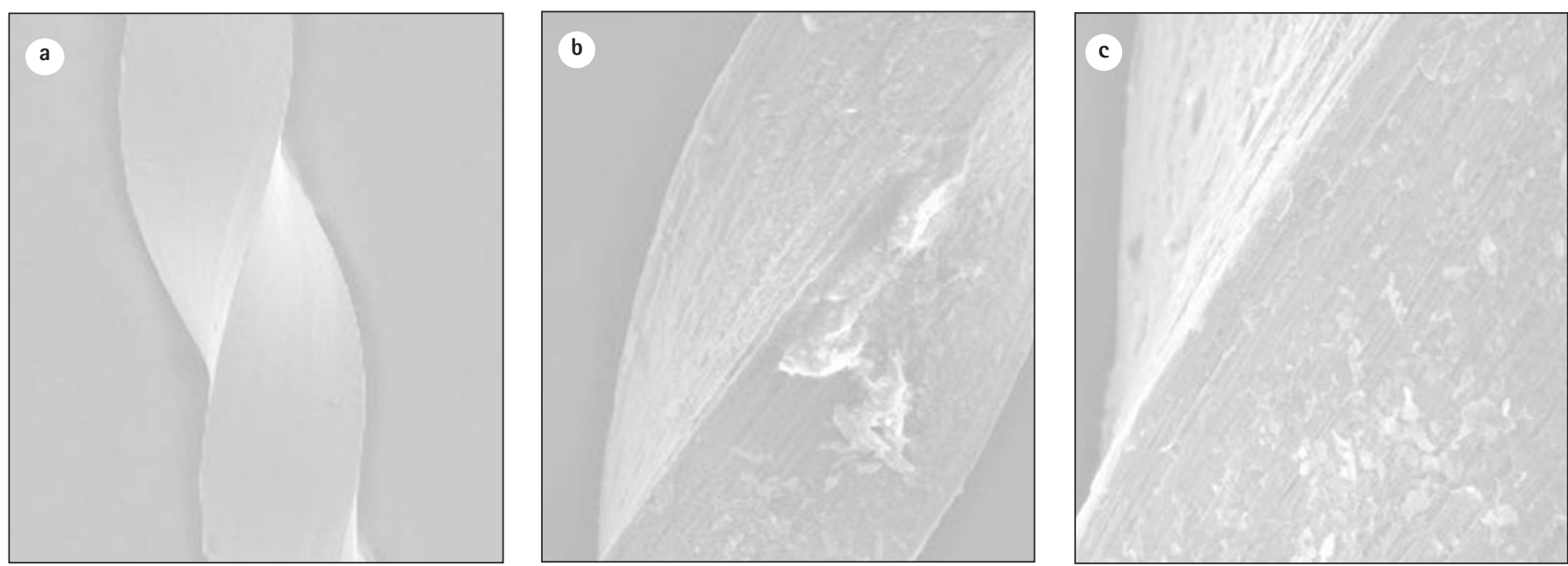

Fig. 18 Illustrations from the work on decontamination of endodontic instruments by Dr Andrew Smith, Glasgow: a) photomicrograph (x16) of an unused endodontic file; b) photomicrograph (x16) of a used instrument after sterilization; c) SEM (x500) of the file shown in illustration b.

\section{Spiral root canal fillers}

Spiral root canal fillers are seldom used in modern endodontics. Their main use is for the insertion of calcium hydroxide into the root canal. When a spiral filler is required, the blade type is preferred by the author, as this is the least likely to fracture. It is essential to ensure that the size selected fits loosely and passively to the required depth before the instrument is rotated in the root canal (Fig. 21).

\section{ROOT CANAL FILLING MATERIALS \\ Gutta-percha}

Gutta-percha is the most commonly used material for the obturation of the prepared root canal system. Standardized gutta-percha points correspond to the ISO sizing system with a $2 \%$ taper. Various other shapes are now available to complement the recently introduced increased taper filing systems (Fig 22). Gutta-percha is the dried resin of the Taban tree, and exists in two forms. Alpha phase is the natural form, but when heated and cooled the beta-phase results. This latter is normally used for root canal filling points.

Gutta-percha points in fact contain only about 20\% gutta-percha. The major component

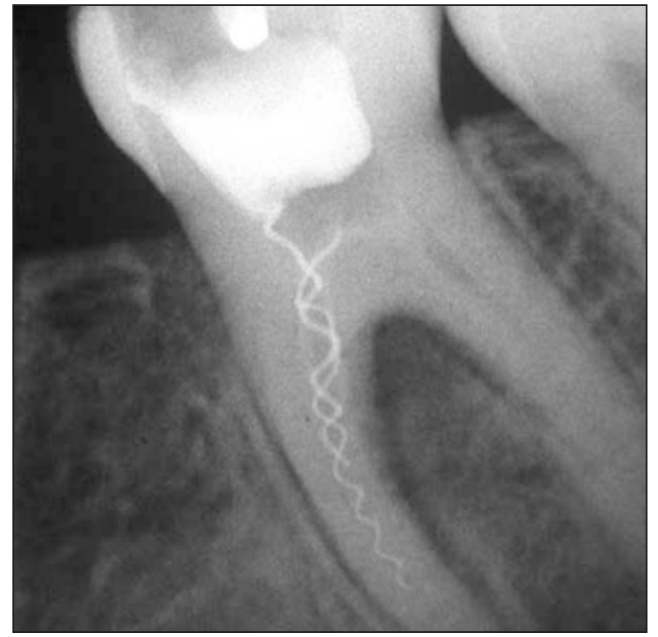

Fig. 21 Spiral fillers may fracture if the size is not verified passively before rotating in the canal. is zinc oxide (up to 75\%), with the remainder comprising various resins, waxes and metallic sulphates to the specific manufacturer's formula.

\section{Sealers/cements}

Root canal sealers play an important role in the obturation of the prepared root canal system, as described in Part 8. Although many proprietary products are available (Fig. 23), they may generally be divided into three groups, according to their main constituents: eugenol, non-eugenol and medicated.

\section{Eugenol}

The eugenol-containing group may be divided into sealers based on the Rickert's formula (1931) and those based on Grossman's (1958) (Table 1). The essential difference between the two groups is that Rickert's contains precipitated silver and Grossman's has a barium or bismuth salt as the radiopacifier. The disadvantage of Rickert's sealer is that the silver will stain dentine a dark grey. One of the most widely used sealers in this group is Tubliseal, a twopaste system and, consequently, simple to mix; it does not contain silver. Tubliseal EWT (extended working time) is preferred.

\section{Non-eugenol sealers}

Some sealers are manufactured with a calcium hydroxide base instead of zinc oxide/eugenol,

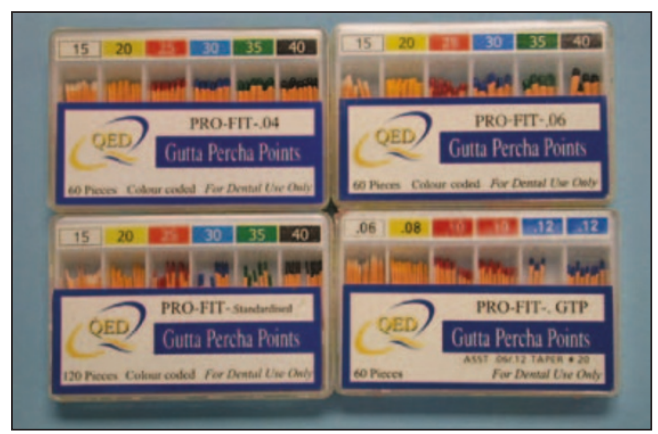

Fig. 22 Some of the different gutta-percha points: standarized; greater taper; 04 and 06 taper; feather tipped.

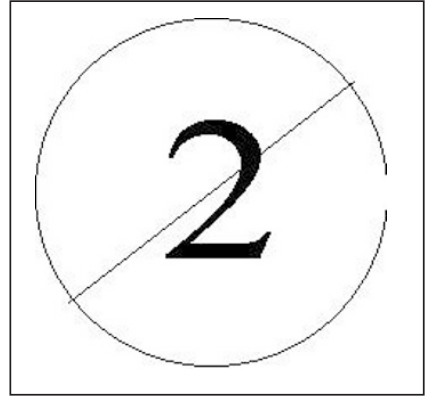

Fig. 19 The symbol indicating instruments intended for single use only.

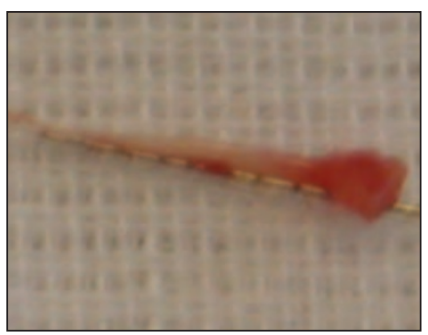

Fig. 20 During root canal treatment of a tooth diagnosed as having an irreversible pulpitis, the vital pulp has been extirpated on a barbed broach. 
Fig. 23 A selection of root canal sealers.
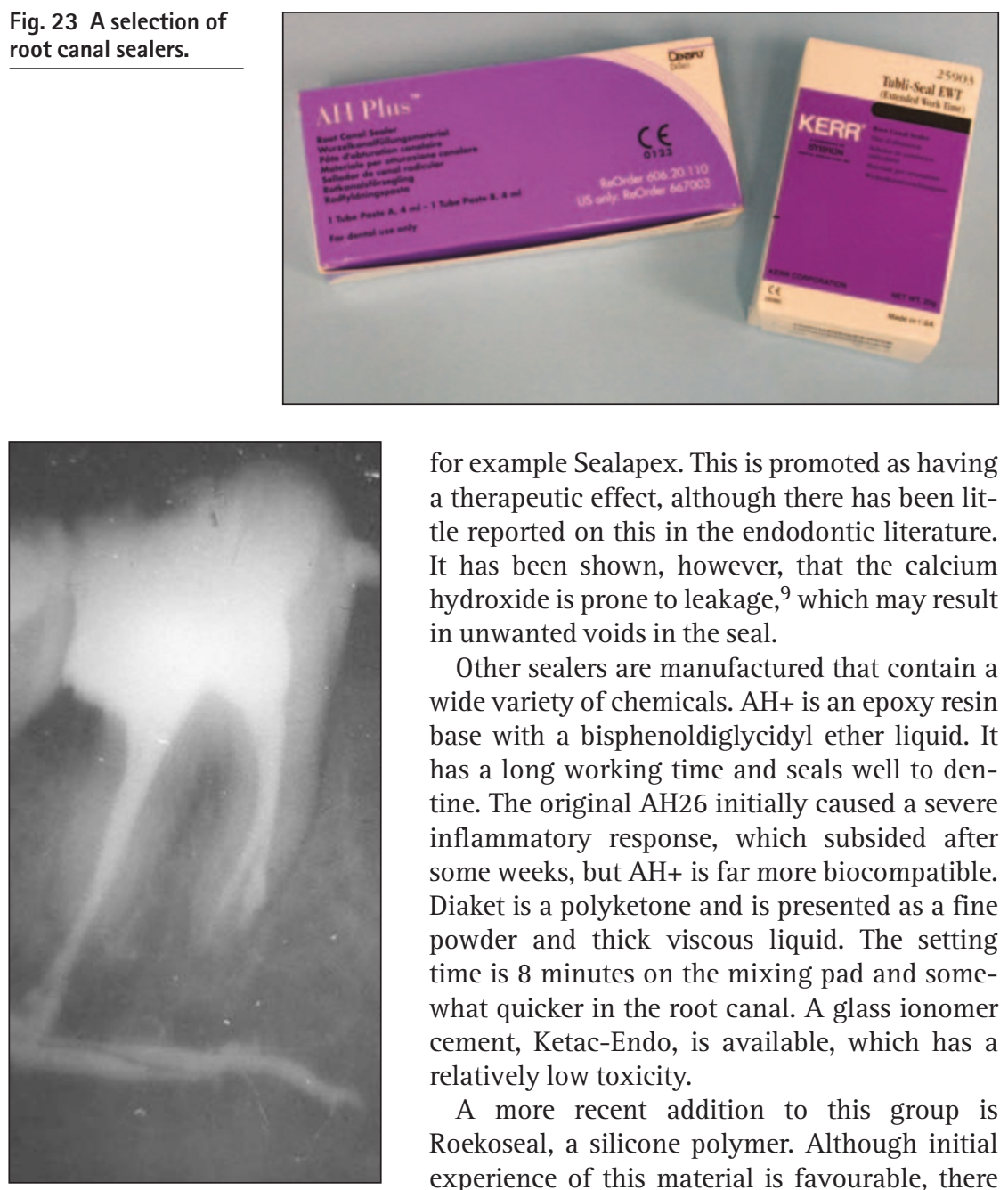

Fig. 24 A medicolegal case where a formaldehyde containing root canal sealer has been extruded into the inferior dental canal, causing paraesthesia of the lip. a therapeutic effect, although there has been litRoekoseal, a silicone polymer. Although initial for example Sealapex. This is promoted as having tle reported on this in the endodontic literature. It has been shown, however, that the calcium hydroxide is prone to leakage, ${ }^{9}$ which may result in unwanted voids in the seal.

Other sealers are manufactured that contain a wide variety of chemicals. $\mathrm{AH}+$ is an epoxy resin base with a bisphenoldiglycidyl ether liquid. It has a long working time and seals well to dentine. The original AH26 initially caused a severe inflammatory response, which subsided after some weeks, but $\mathrm{AH}+$ is far more biocompatible. Diaket is a polyketone and is presented as a fine powder and thick viscous liquid. The setting time is 8 minutes on the mixing pad and somewhat quicker in the root canal. A glass ionomer cement, Ketac-Endo, is available, which has a relatively low toxicity.

A more recent addition to this group is experience of this material is favourable, there has been little published on several of these recent materials, and the prudent clinician may wish to await the results of extended clinical trials before adopting these into their practice.

\section{Medicated}

The current thinking is that provided the principles of root canal preparation and filling are observed, there is no justification for the use of therapeutic sealers. The active ingredient in the majority of medicated sealers is paraformaldehyde, which is usually accompanied by a corticosteroid. Figure 24 shows a medicolegal case where excess medicated sealer entered the inferior dental canal, causing permanent nerve damage with paraesthesia of the lip and soft tissues.

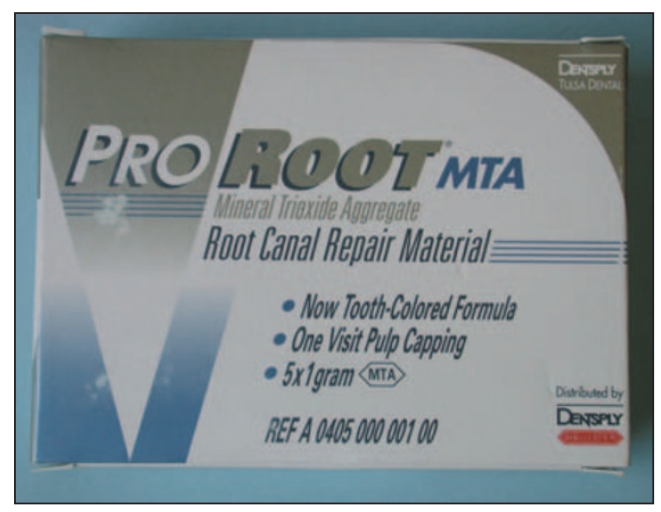

Table 1 Grossman's sealer

\begin{tabular}{ll}
\hline Powder & \\
Zinc Oxide & $42.0 \%$ \\
Staybelite resin & $27.0 \%$ \\
Bismuth subcarbonate & $15.0 \%$ \\
Barium sulfate & $15.0 \%$ \\
Sodium borate (anhydrous) & $1.0 \%$ \\
Liquid & \\
Eugenol & $100 \%$ \\
&
\end{tabular}

\section{Mineral trioxide aggregate}

Mineral trioxide aggregate (Fig. 25) is a compound consisting of mineral oxides, (tricalcium silicate, tricalcium oxide, silicate oxide and traces of other mineral oxides), developed first by Mahmoud Torabinejad and co-workers at Loma Linda University. ${ }^{10}$ Although originally developed as a root-end filling material during periradicular surgery, researchers across the world have reported positive results when the material is used for the repair of perforations, as a pulp capping agent, and to induce apical closure of immature roots. The superb sealing ability, marginal adaptation and biological compatibility of the material appear to make this material the sealant of choice for any communication between the root canal system and the external surface of the tooth. The material is continually being refined, and the latest product has some oxides removed to produce a white, rather than grey, powder. MTA is a difficult material to manipulate, having the consistency of wet sand. Methods of placement are described in Part 11.

\section{Root canal filling instruments Spreaders}

Cold lateral compaction using gutta-percha requires either long-handled or finger spreaders (Fig. 26). These have a long, tapered shank with a sharp point. The instrument is used to compact gutta-percha laterally against the walls of the root canal and provide a space for the insertion of further gutta-percha points. There are several sizes available, and these are selected according to the canal size and the size of the gutta-percha point. The choice of long-handled spreaders or finger spreaders depends on personal preference. The advantage of finger spreaders is that less force can be used, and this reduces the risk of root fracture.

\section{Heat carriers}

The application of heat to the gutta-percha filling permits improved lateral and vertical compaction of the softened material. Ordinary hand and finger spreaders are not designed for this purpose, but the instruments illustrated in Figure 27 may be used. They are of various sizes, and have both a pointed tip for lateral spreading, and a flat tip for vertical compaction.

The instrument shown in Figure 28 is a System $\mathrm{B}$, for the controlled and precise application 
of heat to the gutta-percha filling. Figure 29 shows an Obtura machine, used to deliver heated gutta-percha directly to the root canal. The use of these and other similar machines is described in Part 8.

\section{Magnification}

When asked why endodontics is a difficult subject, undergraduate and postgraduate students alike frequently reply that it is because they cannot see what they are doing. There is no doubt that magnification of the pulp chamber greatly assists in finding and accessing narrow canal orifices, and many practitioners now routinely use loupes, as seen in Figure 30. This one purchase has made huge improvements in the quality and ease of endodontic treatment for many practitioners. Indeed, the improved vision gained from the use of loupes improves all aspects of general dental practice, not just endodontics. The patient in the illustration is merely undergoing a routine examination.

However, specialist practitioners, and some generalists, are moving to the use of surgical microscopes, as seen in Figure 31 where it is being used by a relatively new member of staff in training, who was seeking, and found, a sclerosed canal in an upper incisor.

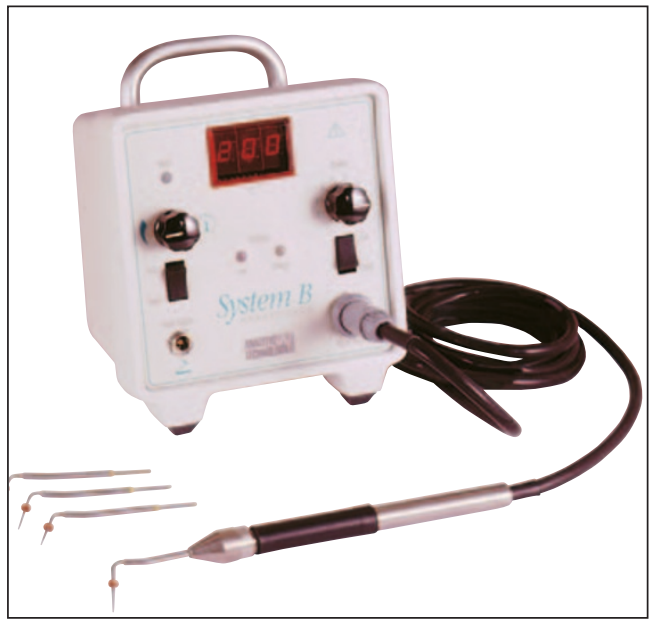

Fig. 28 The System B heat source for controlled warm gutta-percha techniques.

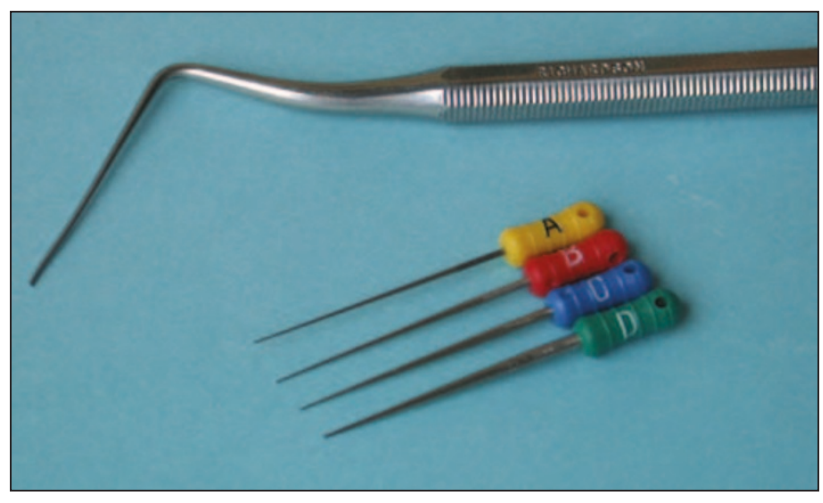

Fig. 26 Cold lateral compaction may be carried out with either finger spreaders or long-handled spreaders.

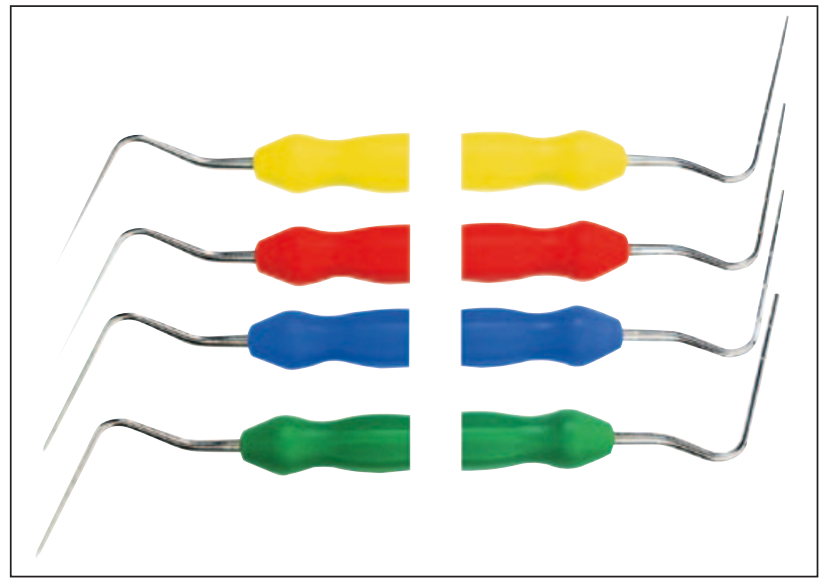

Fig. 27 Machtou heat carrier/pluggers for warm lateral and vertical compaction.

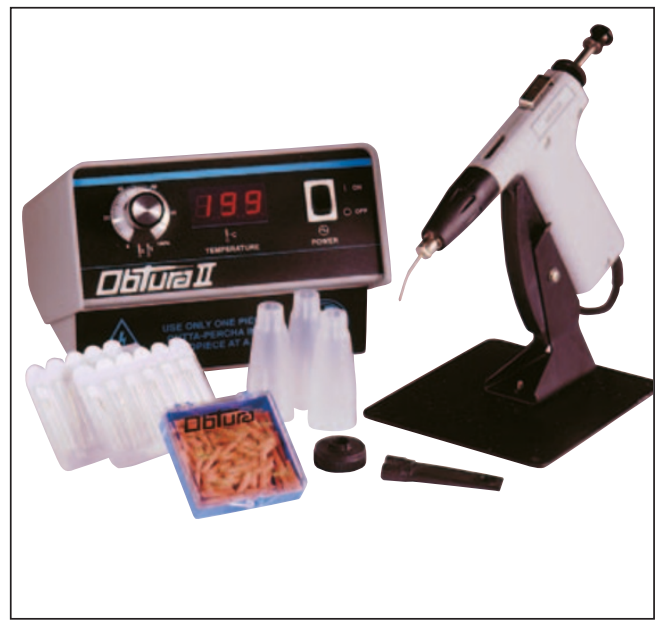

Fig. 29 The Obtura 11 system for injecting heatsoftened gutta-percha into the root canal.

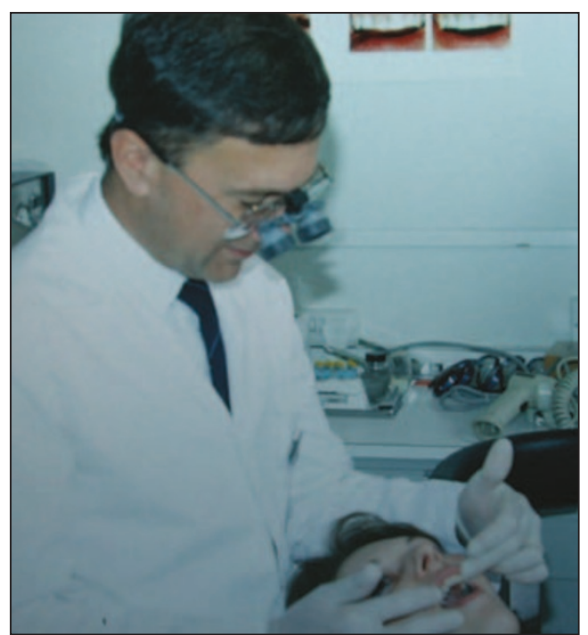

Fig. 30 The use of magnifying loupes is increasing in restorative dentistry.

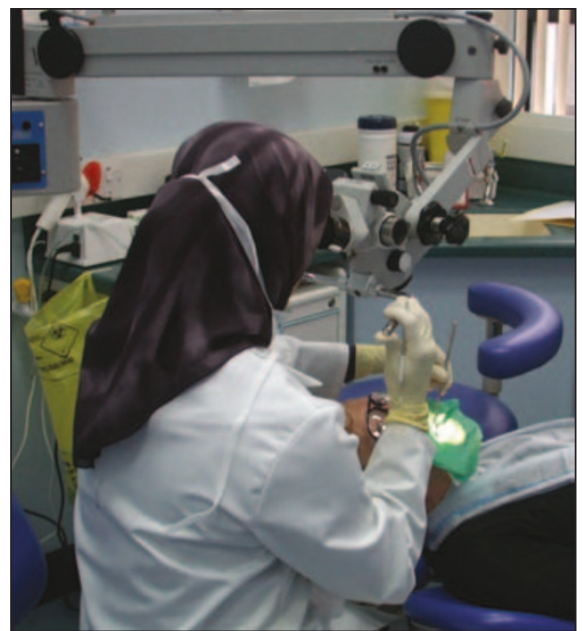

Fig. 31 A surgical microscope may be essential for some of today's intricate endodontic procedures. 
1. National Radiographic Protection Board. Guidance Notes for Dental Practitioners on the safe use of $x$-ray equipment. 2001 Department of Health, London, UK.

2. Cameron J A. The synergistic relationship between ultrasound and sodium hypochlorite: a scanning electron microscope evaluation. J Endod 1987; 13: 541-545.

3. Byström A, Sundqvist G. Bacteriological evaluation of the effect of $0.5 \%$ sodium hypochlorite in endodontic therapy. Oral Surgery, Oral Medicine, Oral Pathology 1983; 55: 307-312.

4. Solovyeva A M, Dummer P M. Cleaning effectiveness of root canal irrigation with electrochemically activated anolyte and catholyte solutions: a pilot study. Int Endod J 2000: 33: 494-504.

5. Haffner C, Benz C, Folwaczny A, Mech A, Hickel R. High frequency current in endodontic therapy; an in-vitro study. J Dent Res 1999; 78: 117.

6. Ranta K, Haapasalo M, Ranta H. Monoinfection of root canals with Pseudomonas aeruginosa. Endod Dent Traumatol 1988; 4: 269-272.

7. Smith A J, Dickson M, Aitken J, Bagg J. Contaminated dental instruments. Journal of Hospital Infection 2002 (in press).

8. Lowe A H, Bagg J, Burke F J T, MacKenzie D, McHugh S. A study of blood contamination of Siqveland matrix bands. BDJ 2002; 192: 43-45.

9. Tronstad L, Barnett F, Flax M. Solubility and biocompatibility of calcium hydroxide-containing root canal sealers. Endod Dent Traumatol 1988; 4: 152-159.

10. Torabinejad M, Hong C U, McDonald F, Pitt Ford T R. Physical and chemical properties of a new root-end filling material. J Endod 1995; 21:349-353. 